\title{
§ 36 Zusammenarbeit der Behörden und Benannten Stellen im Europäischen Wirtschaftsraum und der Europäischen Kommission
}

Die für die Durchführung des Medizinprodukterechts zuständigen Behörden und Benannten Stellen arbeiten mit den zuständigen Behörden und Benannten Stellen der anderen Vertragsstaaten des Abkommens über den Europäischen Wirtschaftsraum und der Europäischen Kommission zusammen und erteilen einander die notwendigen Auskünfte, um eine einheitliche Anwendung der zur Umsetzung der Richtlinien 90/385/EWG, 93/42/EWG und 98/79/EG erlassenen Vorschriften zu erreichen.

Änderungen:

§ 36 Überschr. und Wortlaut geänd. mWv 21.03.2010 durch G v. 29.07.2009 (BGB1. I S. 2326).

1 Die Regelung setzt Art. 20 der Richtlinie 98/79 EG um. Die Zusammenarbeit der Behörden ist im MPG in den $\S \S 15,16,18,25,26,27,29,33$ und 37 angesprochen: Die Zusammenarbeit der Benannten Stellen ist nur in $\S 18$ Abs. 4 erwähnt. Der Daten- und Informationsaustausch erfolgt über das DIMDI und die EUDAMED. 